\title{
Framework for the Energetic Assessment of South and South-East Asia Fixed Chimney Bull's Trench Kiln
}

\author{
Niccolò Le Brun ${ }^{1,2, a}$ and Christos N. Markides ${ }^{2}$ \\ ${ }^{1}$ ThermoCrunch, 144a Albert Palace Mansions, Lurline Gardens, London, SW11 4DJ, United Kingdom \\ ${ }^{2}$ Clea Energy Process (CEP) Laboratory, Department of Chemical Engineering, Imperial College London, South Kensington Campus, \\ London, SW7 2AZ, United Kingdom
}

\begin{abstract}
One of the major sources of fuel consumption and greenhouse gas emission in South and South-East Asia is brick manufacturing. One of the most commonly implemented technologies for brick manufacturing in this region is the fixed chimney Bull's trench kiln (FCBTK). This type of technology largely depends on manual labour and is very inefficient when compared to more modern technologies. Because the adoption of more advanced technologies is hindered by the socio-economical background, the much needed innovations in the brick sector are necessarily related to improving/modifying the FCBTK already operational. However, few scientific studies have been conducted on FCBTK probably due to the basic level of technological development. Such studies are however important to systematically and methodologically assess the challenges and solutions in FCBTK. In this study we develop a thermo-energetic model to evaluate the importance of the parameters pertained to FCBTK construction and operation. The prospective of this study is to build an initial thermo-energetic framework that will serve as a basis to investigate possible energetic improvements.
\end{abstract}

\section{Introduction}

Brick manufacturing in South and South-East Asia is a core economic sector which experienced a rapid expansion in recent years due to the rapid demographic growth and urbanization [1]. Notwithstanding the presence of countries with a developed brick manufacturing industry (e.g. Vietnam), most of the brick production relies on obsolete and inefficient methods which greatly contribute to the countries carbon footprint. In particular, fixed chimney bull's trench kiln (FCBTK) are one of the most implemented type of brick kiln in South and South-East Asia, accounting for more than $70 \%$ of the Indian total brick production [2], and are a considerable source of greenhouse gas emission.

FCBTK generally consists of an approximately $3 \mathrm{~m}$ deep, $8 \mathrm{~m}$ width toroidal trench connected with regular apertures to a fixed structure equipped with a fixed chimney. A sketch of a FCBTK is depicted in Fig. 1. The trench is partially filled with bricks and is then covered with insulation. During operation, green bricks are continuously added by batch in chambers from one end while fired bricks are removed from the other end. Coal (black arrows in Fig. 1) is provided from apertures at the top of the green bricks cluster in a so-called "firing zone", which is normally few meters long. The firing zone is continuously moved so that new green bricks are being fired. The kiln is designed so that the combustion air travels through the fired bricks cooling them; in this way the air is also pre-heated. In addition, the flue gases from

\footnotetext{
${ }^{a}$ Corresponding author: niccolo.le-brun11@imperial.ac.uk
}

the combustion zone pre-heat and dry the green bricks before being conveyed to the chimney. The air/flue gas path is achieved via closing/opening apertures in the fixed kiln structures.

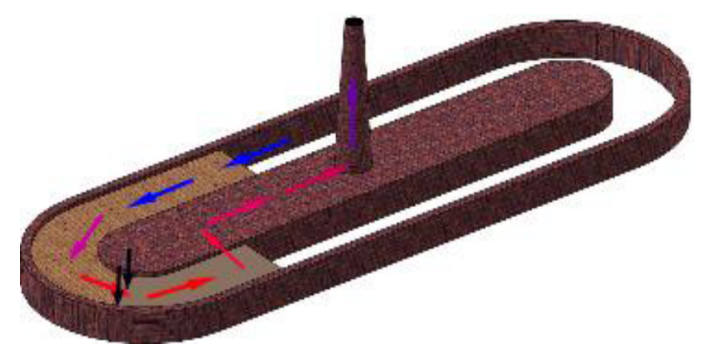

Figure 1. Sketch of a fixed chimney Bull's trench kiln (FCBTK).

Even if FCBTK represents a major step forward compared to the previously adopted, movable chimney bull's trench kiln, they are still far from a satisfactory energy economy. Considering the size of the brick manufacturing industry and the corresponding fossil fuel consumption (second industrial sector for coal consumption in India [3]), new technological developments and best practices will be able to achieve a significant reduction in greenhouse gas emission along with substantially monetary savings [4]. The conversion of FCBTK to zig-zag bull's trench kiln (where the air is conveyed in a zig-zag pattern) is a good example of a 
relatively simple yet important innovation being able to increase both fuel economy and productivity. In order to further improve the efficiency of the FCBTK kiln and reach optimized energetic solutions, it is important to conduct systematic thermo-energetic studies. Apart from the works of Sameer and co-workers [2-3,5], such studies have been neglected in the past years due to the relatively undeveloped nature of FCBTK, which was probably regarded as less attractive than more advanced technologies. As part of this effort, this study proposes to develop a thermo-energetic framework over which the different challenges and the new solutions can be systematically studied.

\section{Model}

The thermal model of FCBTK was developed using a one-dimensional, steady-state, lumped approach where the brick kiln is considered as a continuous process in which the bricks and the surrounding material are progressively being heated by a fictitious stationary firing section, whereas in reality the fire is moved across the stationary bricks. To develop the model, the kiln was assumed as composed of three sections: a pre-heating, a firing and a cooling section. The equations for each section were derived by conducting a mass and energy balance in each section and using simplifying assumptions. The main equations and respective assumptions comprising in the model are listed and discussed in the following lines.

The pre-heating section was modelled as a countercurrent heat exchanger where heat is transferred from the exhaust gas to the green bricks. The brick and gas temperatures were assumed to be uniform over the trench cross-sectional area. Because of the high water content in the green bricks, a constant mass flow-rate of bricks and flue gas could not be assumed during the heating and drying process of the bricks. Instead a spatially dependent mass flow-rate was adopted, as given by the following equation:

$-\frac{\mathrm{d} \dot{m}_{\mathrm{g}}}{\mathrm{d} x}=\frac{\mathrm{d} \dot{m}_{\mathrm{b}}}{\mathrm{d} x}=S_{\mathrm{bg}} k_{\mathrm{bg}}\left(x_{\mathrm{sat}}\left(T_{\mathrm{b}}\right)-x_{\mathrm{g}}\right)$,

where $\dot{m}_{\mathrm{b}}$ is the mass of brick, $S_{\mathrm{bg}}$ is the available surface per unit of length between the bricks and the flue gas, $k_{\mathrm{bg}}$ is the mass heat transfer coefficient, $x_{\text {sat }}$ and $x_{\mathrm{g}}$ are respectively the humidity ratio at the brick surface and in the flue gas. The temperature profile of the bricks and the flue gas in the heating section is found by conducting an energy balance on each stream:

$$
\begin{aligned}
& \left(\dot{m}_{\mathrm{b}}+\dot{m}_{\mathrm{sur}}\right) c_{p, \mathrm{~b}} \frac{\mathrm{d} \tau_{\mathrm{b}}}{\mathrm{d} x}=S_{\mathrm{bg}} h_{\mathrm{bg}}\left(T_{\mathrm{g}}-T_{\mathrm{b}}\right)+ \\
& S_{\mathrm{b} 0} h_{\mathrm{b} 0}\left(T_{0}-T_{\mathrm{b}}\right)-\frac{\mathrm{d} \dot{m}_{\mathrm{b}}}{\mathrm{d} x} \Delta h_{\text {vap }} \\
& \dot{m}_{\mathrm{g}} c_{p, \mathrm{~g}} \frac{\mathrm{d} T_{\mathrm{g}}}{\mathrm{d} x}=-S_{\mathrm{b}} h_{\mathrm{bg}}\left(T_{\mathrm{g}}-T_{\mathrm{b}}\right) .
\end{aligned}
$$

Here $\dot{m}_{\text {sur }}$ is the mass of the surrounding kiln bricks and soil. Neglecting $\dot{m}_{\text {sur }}$ in the model would add a major source of error; indeed as the fire is moved during the kiln operation, new sections of the kiln need to be heated up to temperature, therefore consuming additional energy. The other terms in Eqs. 2-3, namely $c_{\mathrm{pg}}, c_{\mathrm{pg}}, T_{\mathrm{b}}, T_{\mathrm{g}}$ represent the heat capacity and the temperature of respectively bricks and air; $h_{\mathrm{bg}}$ and $h_{\mathrm{bo}}$ represents the brick/flue gas heat transfer coefficient and the brick/environment heat transfer coefficient; $S_{\text {bo }}$ is the brick/environment surface area per unit of length.

The firing section was modelled by considering a uniform firing temperature $T_{\mathrm{f}}$. The energy balance for the solid and gaseous phases can be written as

$$
\begin{aligned}
& \left(\dot{m}_{\mathrm{b}}+\dot{m}_{\mathrm{sur}}\right) c_{\mathrm{p}, \mathrm{b}}\left(T_{\mathrm{b}, \mathrm{in}}-T_{\mathrm{f}}\right)-\dot{m}_{\mathrm{b}} \Delta h_{\mathrm{r}}+ \\
& \dot{m}_{\mathrm{a}} c_{p, \mathrm{a}}\left(T_{\mathrm{a}, \mathrm{in}}-T_{0}\right)-\dot{m}_{\mathrm{g}} c_{p, \mathrm{~g}}\left(T_{\mathrm{f}}-T_{0}\right)- \\
& S_{\mathrm{b} 0} h_{\mathrm{b} 0}\left(T_{0}-T_{\mathrm{f}}\right)+\dot{Q}=0
\end{aligned}
$$

where $Q$ is the heat input rate, related to the amount (rate) of coal consumed. The term $\Delta h_{\mathrm{r}}$ is the enthalpy of reaction and accounts for the energy consumed by the chemical/physical processes during brick firing. The mass of the flue gases is found from the mass of air, $\dot{m}_{\mathrm{a}}$, and the amount of fuel burnt, calculated from the knowledge of the heat input rate $Q$.

The cooling zone is modelled similarly to the heating section, with the mass flow-rate of brick and the mass flow-rate of inlet air assumed constant. The energy balances for this section are:

$$
\begin{aligned}
& \left(\dot{m}_{\mathrm{b}}+\dot{m}_{\mathrm{sur}}\right) c_{\mathrm{p}, \mathrm{b}} \frac{\mathrm{d} T_{\mathrm{b}}}{\mathrm{d} x}=S_{\mathrm{ba}} h_{\mathrm{ba}}\left(T_{\mathrm{a}}-T_{\mathrm{b}}\right)+ \\
& S_{\mathrm{b} 0} h_{\mathrm{b} 0}\left(T_{0}-T_{\mathrm{b}}\right) \\
& \dot{m}_{\mathrm{a}} c_{p_{\mathrm{p}, \mathrm{a}}} \frac{\mathrm{d} T_{\mathrm{a}}}{\mathrm{d} x}=-S_{\mathrm{ba}} h_{\mathrm{ba}}\left(T_{\mathrm{a}}-T_{\mathrm{b}}\right) .
\end{aligned}
$$

The model is completed by giving the expression for the heat transfer coefficients and the mass transfer coefficient, along with the equation governing the combustion process. The complete set of equations was solved in MATLAB using an in-house made algorithm. The algorithm iteratively calculates the heat input rate $Q$ based on the temperature profiles, then finds the mass flow-rate of each stream and finally the temperature profiles. These steps are repeated until convergence. The model was checked for mesh independency and it was found that a 1,000 point spatial discretization gave acceptable results.

\section{Results}

The model was applied to a typical design of a FCBTK. The values of some parameters (such as the mass of surrounding bricks/soil material) were inferred from the study of Sameer [4] which reported a detailed analysis of a Bull's trench kiln. The main values of the model 
parameters are hereby described. The kiln trench was assumed to have a height of $3 \mathrm{~m}$ and a width of $8 \mathrm{~m}$, with an overall length of $130 \mathrm{~m}$. The pre-heating, firing and cooling sections were assumed to have a length of respectively $40,10,80 \mathrm{~m}$. The daily dry brick production was assumed to be $80,000 \mathrm{~kg}$ approximately corresponding to a production of 30,000 bricks per day. The baseline air flow-rate was assumed to be $80,000 \mathrm{~kg} / \mathrm{d}$ of dry air. The amount of coal used varied according to the heat losses and the air flow-rate, with an indicative value of $3,500 \mathrm{~kg} / \mathrm{d}$. The coal was assumed to have a carbon ratio of 0.72 and a lower heating value of $22 \mathrm{MJ} / \mathrm{kg}$. The values of brick-gas/air and bricksurrounding heat transfer coefficients were indicatively between 1 and $10 \mathrm{~W} / \mathrm{m}^{2} \mathrm{~K}$ depending on the different conditions. The mass heat transfer coefficient was assumed to be $0.01 \mathrm{~kg} / \mathrm{m}^{2} \mathrm{~s}$ while the geometric parameters, such as $S_{\text {ba }}$, were calculated based on the standard brick disposition in Bull's trench kilns.

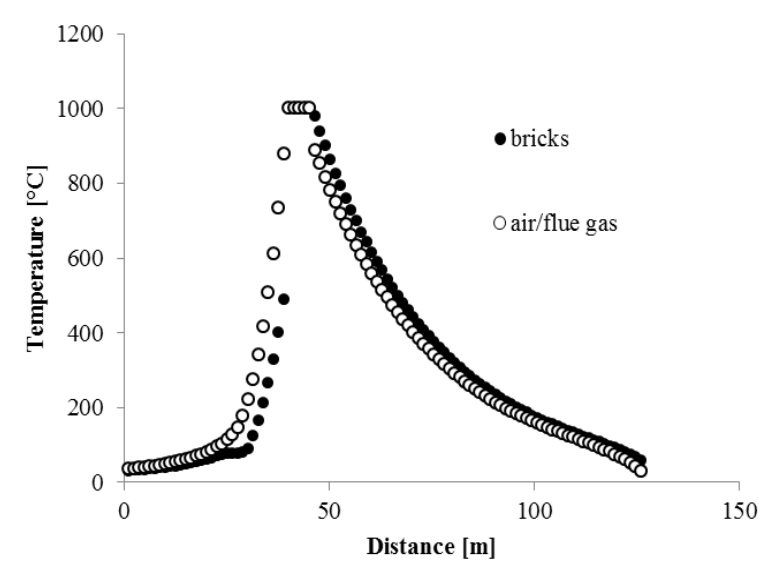

Figure 2. Temperature profiles across the kiln length. The bricks move from left to right in the figure whereas the air/flue gas from right to left. The kiln is divided in a pre-heating section $(0-40 \mathrm{~m})$, a firing section $(40-46 \mathrm{~m})$, and a cooling section $(46-126 \mathrm{~m})$.

The results regarding typical temperature profiles in the pre-heating, firing and cooling regions of the kiln are reported in Fig. 2. It is evident how the temperature of the cooling section decreases more slowly compared to the pre-heating section. The main reason for this behaviour is the lower heat capacity of the air flow compared to the mass of bricks and surrounding material. The mass of surrounding material shifts the heat capacity ratio of the two streams in counter-current flow (hot solid and cold air) from around 1 to more than 1 (around 1.7 in the present case). The lower the heat capacity ratio, the shorter is the distance over which the heat recovery from the hot solid to the cold air takes place. With a higher heat capacity ratio, due to the longer distance exhibiting higher temperatures, the heat losses to the environment from the cooling section are substantial, comprising the largest part of the energy loss. To further understand the effect of air flow-rate, Fig. 3 reports the specific energy consumption (SEC) for different values of excess air and brick-air normalized heat flux, equal to $S_{\mathrm{ba}} h_{\mathrm{ba}}$. As showed, increasing the excess air diminishes the fuel consumption, thus increasing the efficiency. Large amount of excess air $(200-250 \%$, corresponding to an equivalent fuel/oxidant ratio of $0.33-0.28$ ) can achieve a $5-10 \%$ reduction in energy consumption. From the present discussion it follows that the use of a low equivalent ratio (in the range $0.5-0.25$ ) is an intrinsic characteristic of Bull's trench kilns which allows for better heat recovery. Of course, too large amount would results in larger heat losses in the heating section and as sensible heat in the flue gas stream. In Fig. 3, there is an evident increase in SEC for too large amounts of excess air used. This situation was reported in the study of Majumdar et al. [6], in which the major source of heat loss was identified as the sensible heat in the flue gases $(60 \%)$. An accurate tuning of the air flow-rate in the kiln is therefore considered essential to improve the performance of Bull's trench kilns.

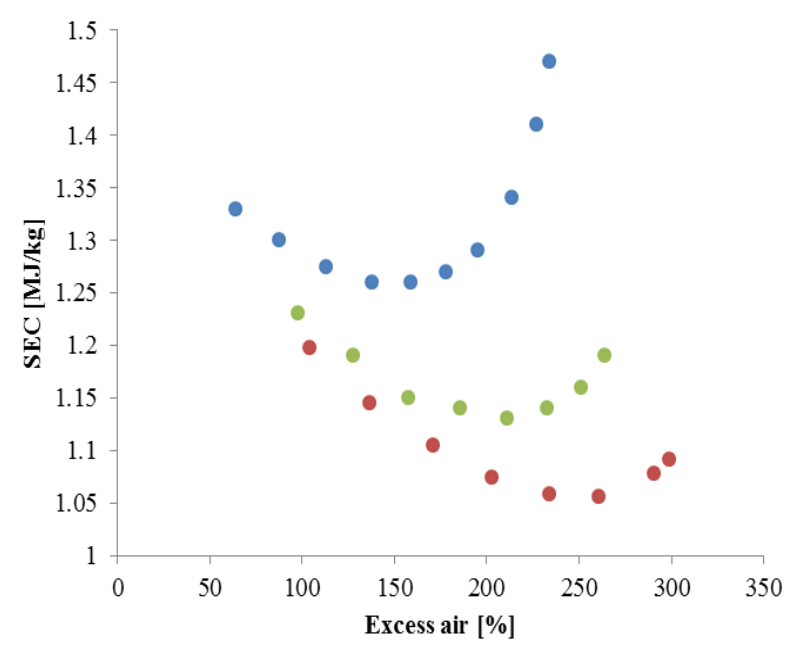

Figure 3. Specific energy consumption (SEC) as a function of the excess air percentage. The different colours refer to different values of $S_{\mathrm{ba}} h_{\mathrm{ba}}: 150 \mathrm{~W} / \mathrm{K}$ (blue), $225 \mathrm{~W} / \mathrm{K}$ (green), and 300 $\mathrm{W} / \mathrm{K}$ (red).

Another possibility to diminish the heat losses to the environment is to increase the brick-air surface and heat transfer coefficient. As showed in Fig. 3, the SEC reduction by increasing the value of $S_{\mathrm{ba}} h_{\mathrm{ba}}$ is substantial. This is the one of the main reasons for the increase in efficiency caused by the conversion from a standard brick arrangement to a zig-zag pattern. The zig-zag flow pattern of the air stream increases both the air-brick surface and the heat transfer coefficient. A higher value of $S_{\mathrm{ba}} h_{\mathrm{ba}}$ leads to a more efficient heat recovery, which in turn decreases the distance over which the temperature is maintained high, decreasing the heat losses to the environment. A zig-zag brick pattern however also increases the pressure losses in the kiln reducing the flow of air. A reduced flow of air could hinder the beneficial effect of the zig-zag pattern as mentioned in the previous discussion. Therefore, in order to fully exploiting the benefits of zig-zag kilns, it seems necessary to increase 
the draft in order to compensate for the larger pressure drops. This can be done by adopting a fan or increasing the height of the chimney. Alternatively, the bricks could be placed in a zig-zag pattern with a less compact arrangement; in this case the pressure drop would be reduced at the expense of productivity.

\section{References}

1. Status and development issues of the brick industry in Asia, Research report, Food and agriculture organization of the united nations, Bangkok, (1993)

2. M. Sameer, D. Lalchandani, G. Malhotra, P. Bhanware, R. Uma, et al., Brick Kilns Performance Assessment, Monitoring of brick kilns \& strategies for cleaner brick production in India, Research Report, (2012)

3. M. Sameer, Evaluating energy conservation potential of brick production in India, Greentech Knowledge Solutions Pvt Ltd, Research report, (2013)

4. Introducing energy-efficient clean technologies in the brick sector of Bangladesh, Research report, Environment, climate change, and water resource unit, South Asia Region, (2011)

5. M. Sameer, Energy utilization in brick kilns, $\mathrm{PhD}$ dissertation, Indian Institute of Technology, Bombay, (2003)

6. Majumdar NC, Ahmed FU, Das K. Survey of thermal efficiency of Bull's trench kilns. Transaction of the Indian Ceramic Society 27, 79-88 (1968) 